\title{
Bandgaps in the propagation and scattering of surface water waves over cylindrical steps
}

\author{
Liang-Shan $\mathrm{Chen}^{1}$, Chao-Hsien $\mathrm{Kuo}^{2}$, Zhen $\mathrm{Ye}^{2}$ * and Xin Sun ${ }^{1,3}$ \\ ${ }^{1}$ Department of Physics, Fudan University, Shanghai 200433, P. R. China \\ ${ }^{2}$ Wave Phenomena Lab, Department of Physics, National Central University, Chungli, Taiwan 32054, R. O. China \\ ${ }^{3}$ National Lab of Infrared Physics, Shanghai Institute of Technical Physics, Shanghai 200083, P. R. China
}

(Dated: July 6, 2018)

\begin{abstract}
Here we investigate the propagation and scattering of surface water waves by arrays of bottommounted cylindrical steps. Both periodic and random arrangements of the steps are considered. The wave transmission through the arrays is computed using the multiple scattering method based upon a recently derived formulation. For the periodic case, the results are compared to the band structure calculation. We demonstrate that complete band gaps can be obtained in such a system. Furthermore, we show that the randomization of the location of the steps can significantly reduce the transmission of water waves. Comparison with other systems is also discussed.

PACS numbers: $47.10 .+\mathrm{g}, 47.11 .+\mathrm{j}$
\end{abstract}

Phenomena pertinent to waves in complex media have been and continue to be a great inspiration for the development of scientific explorations. One of the most important phenomena is frequency band structures. It prevails when waves propagate through periodically structured media. Such a phenomenon was first investigated for electrons in solids nearly eighty years ago. The wellknown Bloch theorem has then been proposed and led to the successful explanation of such important properties of solids as conductivity, semi-conductivity, and insulating states [1]. Applying these concepts to classical waves 2, 3] has paved an avenue to the new era of research. Not only all the phenomena previously observed or discussed only for electronic systems are successfully transplanted to classical systems, but many more significant and novel ideas and applications, such as photoniccrystal-made negatively-refractive devices [4], have well gone beyond expectation, and are so far reaching that a fruitful new field has been established, $i$. e e the field of photonic and acoustic crystals, signified by the establishment of a comprehensive archive website [5].

Recently, the consideration of waves in periodic media has also been deliberately extended to the propagation of water waves over periodically structured bottoms 6 , 7, 8, 9, 10. Some of the advances have been reviewed, for example, by McIver 11]. One of the most recent pioneering experiments used water waves to illustrate the phenomenon of Bloch waves as a result of the modulation by periodic bottom structures [9]. This experiment made it possible that the abstract concept be presented in an unprecedentedly clear manner.

Motivated by the experiments [9], in this paper we would like to further explore the propagation of water waves through underwater structures. The structures considered here consist of arrays of cylindrical steps mounted on a flat bottom. Although there have been many theoretical approaches for investigating propagation of water waves over various bottom topographies, most approaches have been limited to mildly varying bottom structures or their variations 12, 13. In this paper, we will use the recently derived theory 14 . The main reason lies in that the theory was successfully in explaining the experimental observations 15]. In addition, it has been shown that this approach compares favorably with existing approximations when applied to some special cases considered previously. We will calculate the wave transmission and band structures for periodically arranged arrays. Then we will show the effect of positional disorders on the transmission. The results suggest that the phenomenon of complete band gaps by analogy with the photonic crystals is also possible for water waves. The complete band gaps refer to the frequency regime where waves cannot propagate in any direction.

Before continuing, it is worth noting here that from a more general perspective, propagation of water waves over topographical bottoms has been a subject of much research from both practical and theoretical aspects since Lamb 16]. From the practical side, the topic is essential to many important ocean engineering problems such as floating bridges and devices in offshore power stations 11] A great amount of papers and monographs has been published 17, 18, 19, 20, 21, 22, 23, 24, 25]. A comprehensive reference on the topic can be found in two excellent textbooks 12, 13.

A conceptual layout of the system considered here is presented in Fig. 11 The water surface is in the $x-y$ plane. Governing equations for the motion of surface water waves in such a system can be obtained by invoking the Newton's second law and the conservation of mass by assuming that the water is incompressible. While the detailed derivation has been given elsewhere [14], here we just list the final equations.

The displacement of the water surface is denoted by $\eta(\vec{r}, t)$. Its Fourier transformation is

$$
\eta(\vec{r}, t)=\frac{1}{2 \pi} \int_{-\infty}^{\infty} d \omega e^{-i \omega t} \eta_{\omega}(\vec{r})
$$




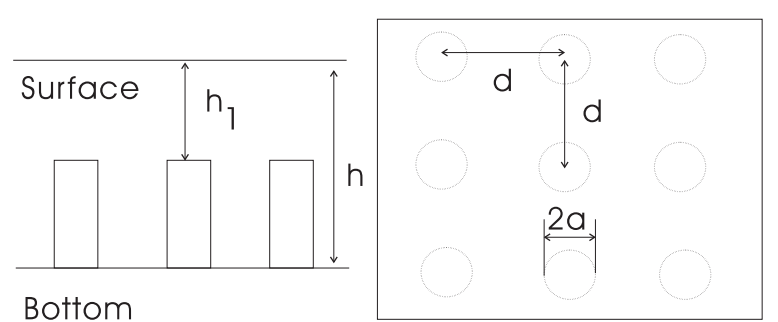

(a)

(b)

FIG. 1: Conceptual layout of the system: (a) side view; (b) bird's view. Here the cylindrical steps with height $\Delta h=h-h_{1}$ form a rectangular array with lattice constant $d$.

The equation of motion for the Fourier component $\eta_{\omega}$ is derived as 14.

$$
\nabla\left(\frac{1}{k^{2}} \nabla \eta_{\omega}(\vec{r})\right)+\eta_{\omega}(\vec{r})=0,
$$

where $\nabla=\partial_{x} \vec{e}_{x}+\partial_{y} \vec{e}_{y}$, and the wavenumber $k$ satisfies

$$
\omega^{2}=g k(\vec{r}) \tanh (k(\vec{r}) h(\vec{r})) .
$$

For a fixed frequency $\omega$, the wavenumber varies as a function of the depth $h(\vec{r})$.

Although Eq. (2) can be used for arbitrary topographical bottoms, in this paper we focus on the cylindrical steps depicted in Fig. 11 Furthermore, we assume that all the steps are identical. When there is a stimulating source, the transmitted waves will be scattered repeatedly at the steps, forming an orchestral pattern of multiple scattering. Such a multiple scattering process can be solved for any arrangement of the steps with help from the theory devised by Twersky 26. The wave transmission can be computed. In the computation, the transmission is normalized such that it is unity when there are no scatterers. The details have been presented in [14].

When the steps are regularly placed to form periodic lattices, the frequency bands will appear and can be determine as follows. By Bloch's theorem [1], the displacement field $\eta_{\omega}$ can be expressed in the following form

$$
\eta_{\omega}(\vec{r})=e^{i \vec{K} \cdot \vec{r}} \sum_{\vec{G}} C_{\omega}(\vec{G}, \vec{K}) e^{i \vec{G} \cdot \vec{r}}
$$

where $\vec{G}$ is the vector in the reciprocal lattice and $\vec{K}$ the Bloch vector [1]. In this case, the wavenumber $k$ also varies periodically and we have the following expression

$$
\frac{1}{k^{2}}=\sum_{\vec{G}} A_{\omega}(\vec{G}) e^{i \vec{G} \cdot \vec{r}}
$$

For a fixed $\omega$, the coefficients $A_{\omega}$ are determined from Eqs. (3) and (5).
Substituting Eqs. (4) and (5) into Eq. (2), we get

$$
\sum_{\vec{G}^{\prime}} Q_{\vec{G}, \vec{G}^{\prime}}(\vec{K}, \omega) C_{\omega}\left(\vec{G}^{\prime}, \vec{K}\right)=0,
$$

with

$Q_{\vec{G}, \vec{G}^{\prime}}(\vec{K}, \omega)=\left[(\vec{G}+\vec{K}) \cdot\left(\vec{G}^{\prime}+\vec{K}\right)\right] A_{\omega}\left(\vec{G}-\vec{G}^{\prime}\right)-\delta_{\vec{G}, \vec{G}^{\prime}}$.

The dispersion relation connecting $\vec{K}$ and $\omega, i$. e. the frequency bands, is therefore determined by the secular equation

$$
\left.\operatorname{det}\left[(\vec{G}+\vec{K}) \cdot\left(\vec{G}^{\prime}+\vec{K}\right)\right] A_{\omega}\left(\vec{G}-\vec{G}^{\prime}\right)-\delta_{\vec{G}, \vec{G}^{\prime}}\right]_{\vec{G}, \vec{G}^{\prime}}=0 .
$$

Special care has to be taken in solving the above secular equation, since the initial dispersion relation in Eq. (3) is non-linear. We use an iterative procedure to find the zero point or the fixed point for the determinant. To gain confidence with the computation, we have applied the numerical codes to three special cases for which the solution is relatively easy to obtain: a flat bottom, steps in shallow and deep waters. We found that the results match well the expectations.

First we consider the case of regular arrays. Figure 2 shows the results for the band structures and the transmission of water waves across the arrays of cylindrical steps. With reference to [9], the following parameters have been used in the computation: lattice constant $\mathrm{d}=$ $2.5 \mathrm{~mm}$, cylinder radius $\mathrm{a}=0.875 \mathrm{~mm}$, depth of the water $\mathrm{h}=2.5 \mathrm{~mm}$. The heights for the steps are 2.49 and $2.40 \mathrm{~mm}$ for (a) and (b) respectively. When computing the transmission, a stimulating source is placed about one lattice constant away from the arrays whereas the receiver is located at about half of the lattice constant away on the other side of the arrays. To ensure the stability of the results, enough modes and number of steps have been considered. For instance, the maximum mode number and the maximum array size considered are 9 and $30 \times 10$ respectively. These numbers are considerably larger than what has been computed previously.

Here it is shown that there is a complete band gap ranging from 9.6 to $12.3 \mathrm{~Hz}$ for the case in (a). The band structure calculation in(a1) is fully supported by the independent transmission calculation by the multiple scattering theory. Along the $\Gamma X$ direction, the band structure shows that there is a partial frequency gap from about 15 to $22 \mathrm{~Hz}$. That is, waves whose frequency lies within the range cannot propagate along this direction. This partial gap also appears in the transmission calculation shown by the solid line in (a2). However, the gap depicted by the transmission calculation seems much narrower than that obtained by the band structure calculation. We find that this is due to the finiteness of the array. Reasons follows. Since a point source was used to transmit waves, waves can be radiated to various direction. Although the propagation along the $\Gamma X$ direction 

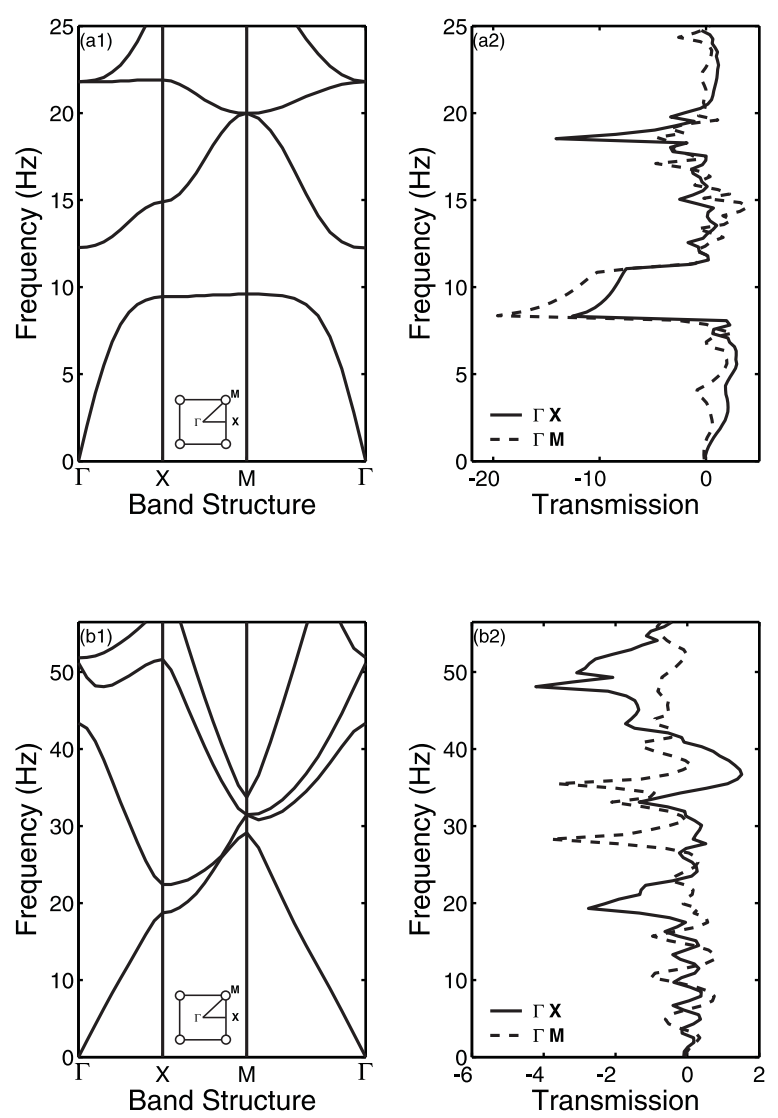

FIG. 2: Right Panel: Normalized transmission $\ln |T|^{2} \mid$ versus frequency for square lattices cylindrical steps with two deferent heights. Left panel: the calculated band structures for the corresponding lattices. The inserted boxes in (a1) and (b1) denote the Brillouin zone and illustrates the direction of wave transmission. For example, $\Gamma X$ and $\Gamma M$ refer to [10] and [11] direction respectively.

is prohibited in the presence of the partial gap, the radiation into other directions may still have the chance to arrive at the receiver, thus complicating the observation of the partial gap.

We also find that the band structures and the transmission are very sensitive to the arrangement and the height of the steps. As an example, in Fig. 2(b) we show the results for the same lattice array as in (a) except that we change the height of the steps from 2.49 $\mathrm{mm}$ to $2.40 \mathrm{~mm}$. This slight change causes a dramatic change in band structure. The complete band gap disappeared. There are two partial gaps located at 20 and 45 $\mathrm{Hz}$ respectively along the $\Gamma X$ direction. Within these two gaps, the transmission is inhibited, as evidenced by the two leftward valleys on the solid line in (b2). Compared to the situation in (a), the transmission data match the band structures better for the partial gaps.

We notice that there are two inhibited transmission valleys along the $\Gamma M$ direction from the multiple scattering calculation, referring to the dotted line in (b2).
This phenomenon is surprising, since in the frequency range concerned, roughly from 28 to $35 \mathrm{~Hz}$, two frequency bands do show up in diagram (b1). A possible explanation for this ambiguity may be that the two bands are deaf. Such a deaf-band phenomenon has been recently observed, for example, in acoustic systems 27 with a further support from theoretical computations 28$]$.

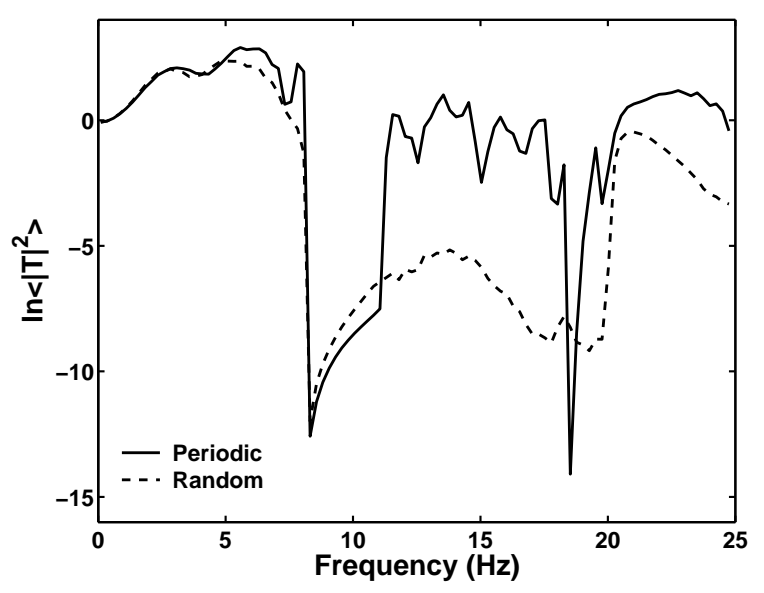

FIG. 3: Normalized transmission $\ln |T|^{2}$ versus frequency for periodic and complete random arrays respectively. In the random case, the transmission has been averaged over the random configurations.

Next we consider the effect of the randomization in the locations of the steps. For brevity, we only show the result for the complete random array. That is, the locations of the cylindrical steps are completely random on the $x-y$ plane. The only restraints are that no two steps should overlap with each other, and the averaged distance between two nearest steps is kept as the same as in the ordered case, i. e. $d=2.5 \mathrm{~mm}$. The transmission results are shown in Fig. 3 The solid and dotted lines separately refer to the transmission results for the propagation along the [10] direction in the ordered case and for the complete random case. At low frequencies, the disorder effect is not obvious for the given sample size. In this regime, the scattering by the steps is week. However, the transmission is significantly reduced in the mid range of frequency. This observation is in agreement with the case of acoustic scattering by arrays of rigid cylinders located in air 14]. For high frequencies, the reduction due to the disorder is not as significant. This is understandable. In the high frequency range, for example when $k h>>1$, the effect of the steps on the wave propagation tends to diminish. This can be seen from Eq. (3) which reduces to $\omega^{2} \approx g k$ in the high frequency regime.

In summary, we have considered water wave propagation over bottom-mounted cylindrical steps. We found that complete band gaps can appear in such a system in analogy with that in the photonic or sonic crystals. The results also suggest that there might be deaf-band 
phenomenon for the water waves.

Acknowledgements The work received support from Fudan University, NSC and NCU. One of us (YZ) is grateful to the government of Shanghai for the Bai YuLan fund which makes his visit to Fudan University possible. Useful help, discussion and correspondence with Profs. J.-H. He, P. G. Luan, P. McIver, and J. Zi are also thanked.

* Electronic address: zhen@phy.ncu.edu.tw

[1] N. W. Ascroft and N. D. Mermin, Solid State Physics (Saunders College, Philadelphia, 1976).

[2] L. Brillouin, Wave Propagation in Periodic Structures, (McGraw-Hill, New York, 1946).

[3] E. Yablonovitch, Phys. Rev. Lett. 58, 2059 (1987); S. John Phys. Rev. Lett. 58, 2486 (1987).

[4] C. Luo, S. G. Johnson, D. J. Joannopoulos, and J. B. Pendry, Phys. Rev. B 65, 201104(R) (2002); H. Kosaka, T. Kawashima, A. Tomita, M. Notomi and T. Tamamura, T. Sato and S. Kawakami, Phys. Rev. B 58, R10096 (1998).

[5] http://www.pbglink.com

[6] T. J. O'Hare and A. G. Davies, Appl. Ocean Res. 15, 1 (1993).

[7] T. Chou, Phys. Rev. Lett. 79, 4802 (1997); ibid, J. Fluid Mech. 369, 333 (1998).

[8] D. V. Evans and R. Porter, J. Eng. Math. 35, 149 (1999); R. Porter and D. V. Evans, J. Fluid Mech. 386, 233 (1999).
[9] M. Torres, J. P. Adrados, F. R. Montero de Espinosa, Nature 398, 114 (1999).

[10] Y.-K. Ha, J.-E. Kim, H.-Y. Partk, and I.-W. Lee, Appl. Phys. Lett. 81, 1341 (2002).

[11] P. McIver, Appl. Ocean Res. 24, 121 (2002).

[12] C.-C. Mei, The Applied Dynamics of Ocean Surface Waves, (World Scientific, Singapore, 1989).

[13] M. W. Dingemans, Water Wave Propagation over Uneven Bottoms, (World Scientific, Singapore, 1997).

[14] Z. Ye, Phys. Rev. E 67, 036623 (2003).

[15] M. Torres, et al., Phys. Rev. E 63, 011204 (2000).

[16] H. Lamb, Hydrodynamics, (Dover, New York, 1932).

[17] M. S. Longuet-Higgins, J. Fluid Mech. 1, 163 (1956).

[18] E. F. Bartholomeuz, Proc. Cambridge Philos. Soc. 54, 106 (1958).

[19] J. W. Miles, J. Fluid Mech. 28, 755 (1967).

[20] D. H. Peregrine, Adv. Appl. Mech. 16, 9 (1979).

[21] R. E. Meyer, Adv. Appl. Mech. 19, 53 (1979).

[22] H. Kagemoto and D. K.-P. Yue, J. Fluid Mech. 166, 189 (1986).

[23] P. Devillard, F. Dunlop, and B. Souillard, J. Fluid Mech. 186, 521 (1988); M. Belzons, E. Guazzelli, and O. Parodi, J. Fluid Mech. 186, 539 (1988).

[24] C. M. Linton and D. V. Evans, J. Fluid Mech. 215, 549 (1990).

[25] J. N. Newman, J. Fluid Mech. 23, 399 (1965).

[26] V. Twersky, J. Acoust. Soc. Am. 24, 42 (1951).

[27] J. V. Sánchez-Pérez, et al, Phys. Rev. Lett. 80, 5325 (1998).

[28] Y.-Y. Chen and Z. Ye, Phys. Rev. Lett. 87, 184301 (2001). 